\title{
Ludwig Bach †
}

Nur wenige Monate erst sind ins Land gegangen, seit sieh dieGruft über den sterblichen Resten J. v. Michels geschlossen, und schon raffte ein tragisehes Geschick den einzigen akademischen Faehvertreter seiner Schule, den Geh. Med.-Rat Professor Dr. Ludwig Bach, hinweg.

Wer von den Teilnehmern an der letzten Zusammenkunft der Heidelberger Ophthalmologischen Gesellschaft hätte es auch nur ahnen können, daß er schon so bald seinem Meister nachfolgen würde, dessen schon gebrochene Gestalt, der Stütze bedürftig, sieh auf den starken Arm des Jüngers lehnte, als er das Rednerpult betrat, urn seine letzten Worte an die versammelten Faehvertreter zu richten!

Im Alter von erst 46 Jahren, in der Vollkraft des Mannes und auf der Höhe seines Wirkens, mußte Ludwig Bach von uns gehen, ein ernstes Memento für alle, die da schaffen und hoffen. Ihm war es nicht ver-gönnt, die Früchte dessen zu ernten, was er in barter Arbeit gesät hatte. Daß er aber nicht umsonst gelebt, werden die folgenden Zeilen zu be-weisen suchen.

Ludwig Bach wurde am 31. Dezember 1865 zu Frankweiler in der Rheinpfalz als Sohn eines Weingutsbesitzers geboren. Seine erste

564 Ludwig Bach $\dagger$

Ausbildung erhielt er auf dem humanistischen Gymnasium zu Landau, nacli dessen Absolvierung er 1884 die Universität München bezog, um sich dem Studium der Medizin zu widmen, das er zunäehst in Berlin und später in Würzburg fortsetzte. Hier erfolgte 1888 seine Promotion zum Dr. med., und bestand er ein Jahr später das ärztliche Staats-examen. Darauf hielt er sich zur weiteren Ausbildung wiederum in München und Berlin auf, kehrte dann nach Würzburg zurück und war dort von 1881 bis 1900 Assistent an der Universitäts-Augenklinik unter J. v. Michel. In das Jahr 1894 fällt seine Habilitation als Privatdozent für das Fach der Ophthalmologie an der Würzburger Universität. Im Jahre 1900 wurde Bach als o. ö. Professor der Augenheilkunde an die Universität Marburg berufen und gieichzeitig mit der Direktion der dortigen Augenklinik betraut. Hier blieb er bis zu seinem frühen Ende.

öanz unerwartet erfüllte sich sein Geschick, und noch heute fällt es uns, seinen engeren Schülern, schwer, das fast Unfaßbare zu begreifen, Seine Erkrankung begann mit einem scheinbar leichten Gesichtserysipel, mit dem sich der Yerstorbene höchstwahrscheinlich in Ausübung seines Berufes infiziert hatte. Mit Intermissionen zeigte das Leiden den üblichen Verlauf und befiel in sprungweisem Fortschreiten allmählich die ganze linke Gesichtshälfte. Eigentlich bedrohlich waren die Er-scheinungen nie, und am Nachmittage des 9. Mai schien nach etwa achttägigem Krankenlager begründete Hoffnung auf einen baldigen glücklichen Ausgang vorhanden zu sein. Da erfolgte ganz plötzlich und unerwartet der fux'chtbare Schlag. Der Kranke schlummerte gegen Abend ein und erwachte nochmals kurz gegen Mitternacht, um dann, der drohenden Gefahr seiner Lage zum Glücke nicht bewußt, in den ewigen Schlai zu verfallen. Die Krankenschwester bemerkte in der frühen Morgenstunde des 10. Mai einen jähen und unheimlichen Wechsel im Aussehen des Kranken, was sie veranlaßte, sofort die Angehörigen zu benachrichtigen und ärztliche Hilfe herbei-zuholen. Der Zustand erschien denn auch äußerst bedrohlich; tiefes Coma, Lungenödem, schlechte Herztätigkeit und schwere Xephritis 
beherrschten das Krankheitsbild, und es blieb nur wenig Hoffnung auf Aenderung zum Besseren, als es im Laufe des Tages trotz Anwendung aller erdenklichen Mittel nicht gehngen wollte, die völlig darnieder-liegende Nierentätigkeit wieder anzuregen. Im tiefsten urämischen $(1 / 8 \mathrm{ma}$ erfolgte gegen die 10. Abendstunde der befürchtete Ausgang, ein entsetzlicher Schlag für seine Familie - seine Gattin und drei noch unmündige Kinder betrauern den Gatten und Vater -, seine Freunde, Kollegen und Schüler. Die sterblichen Ueberreste wurden mit alien akademischen Ehren hinausbegleitet und am Nachmittage desselben Tages in Mainz dem Wunsche des Entschlafenen entsprechend eingeäschert. Seine Asche soil auf dem Marburger Friedhofe beigesetzt $\mathrm{w}$ Гerden.

Elf Jahre war es mir vergònnt, mit Ludwig Bach zusammen in Marburg tätig zu sein, ich habe seine Entwicklung und die der von ihm geleiteten Klinik verfolgen können und bin daher gern der Aufforderung nachgekommen, ihm einige herzlich und ernst empfundene Worte übers Grab hinaus nachzurufen.

Ludwig Bach $\uparrow$. $\quad 565$

Es ergibt sich aus der berufliehen Stellung des akademischen Klinikers von selbst, daß man seine Bedexxtxxng nach dem zu beurteilen hat, was er als Forscher, Lehrer xxixd Arzt leistete. Und es scheint mir angemessen und dem Entwicklungsgange Ludwig Backs entsprechend xxx sein, den Buck des Lesers zuerst axxf seine Forschertätigkeit zu lenken, die sich naturgemäß hauptsächlieh im Rahmen seines ophthal-mologischeix Spezialfaehes bewegt hat, ohne jedoch den Zusammenhang in it der gesamten Heilkunde je zxx verlieren. Auf seinem Gebiete hat er allerdings eine Arbeit geleistet, wie sie iix so kurzer Zeit nur wenigen vergönnt war, und es gibt kaum ein Einzelgebiet in der Augenheilkunde, auf dem er nicht wenigstens Spxxren seiner wissenschaftlichen Tätigkeit zurückläßt.

Seine ersten in Würzburg entstandenen Arbeiten, mehr kasuistischen Inhaltes, behandelten Fälle von Störungen der Augenmuskelfunktion, Lidbewegung, Nystagmus etc. Allein derartige Arbeiten konnten sein drängendes Bedürfnis nach wissenschaftlicher Betätigung nicht befriedigen, und schon frühzeitig ging Bach daran, einzelne umgrenzte Gebiete der ophthalmologischen Disziplin von bestimmten Gesichts-punkten aus einer gründlichen Nexxbearbeitung zu xxnterziehen.

Es waren zuerst anatomische Stxxdien an der menschlichen Xetz-haut, deren Ganglienzellenstruktur er mit modernen Untersuchungs-methoden durchforschte, dann Arbeiten über die Gefäße und Nerven der Lider, der Hornhaut und der Sklera des Menschen und verschiedener Tiere. Dem folgten Mitteilungen axxs dem Gebiete der Mißbildungen des menschlichen Auges, verschiedener Starformen, der Colobome des Bulbus, sowie der angeborenen Anomalien der Pxxpillenform, speziell der Korektopie. Weiterhin beschäftigte ihn die Frage nach dem Zustandekommen der sympathischen Ophthalmie, die er auf experimentellem und klinischem Wege zu beantworten versuehte. Er kam zu der Axiffassung, daß den Ciliarnerven als Yermittlern der sympathischen Erkrankung doch wohl eine größere, $\lambda$ venn auch mehr indirekte Rolle beim Zustandekommen dieses in seiner Genese auch heute noch viel xunstrittenen Krankheitsbildes zukomme, xxnd von dieser seiner Auf-fassung hat er sich trotz aller experimentellen Gegenbeweise nicht frei-machen können. Wer will entscheiden, ob nicht vielleicht mit Recht!

Dann $\lambda$ vandte er seine Aufmerksamkeit bakteriologischen Studien zu und bemühte sich, in die damals xxoch etvvas verworrenen Vor-stellungen vom Keimgehalte des Bixxdehautsackes, der bakteriziden Kraft der Tränenflüssigkeit, sowie von Asepsis und Antisepsis in der Augenheilkunde Klarheit zu bringen. Seine Untersuchungen dienten vor allem dem 
Haxxptzwecke der Erforschung der besten und erfolg-reichsten Art der praktischen Wundbehandlung, speziell nach Star-operationen; xxnd seiner Auffassung, daß nicht durch antiseptisches, sondern durch aseptisches Operieren die besten Resultate erzielt würden, hat er auch später auf Grund eingehender, an seiner eigenen Klinik angestellter Versuche noch oft beredte Worte verliehen. Aus persön-licher Erfahrung kann ich hinzufügen: mit Recht! Denn eine ein-fache Durchspülung des Bindehautsackes mit steriler physiologischer Koehsalzlösung xmd entsprechende schonende Reinigung der Lidränder

566 Ludwig Bach $\uparrow$.

hat unter gleichzeitiger Beachtung strengster Asepsis bei der Star-operation genügt, die Zahl der postoperativen Infektionen auf ein ver-schwindeixdes Mindestmaß herabzudrücken.

In $\lambda$ veiteren bakteriologisch-klinischen Arbeiten behandelte der Ge-lehrte die txxberkxxlöse Infektion des Auges und seiner Adnexe, wozu ihm die Klientel der Würzburger Augenklinik ein besonders reichliches und interessantes Material bot. Dabei sehenkte er der Frage nach dem Zusammenhange der ekzeniatösen Erkrankungen mit deix sicher tuber -kixlösen besondere Aufmerksarnkeit.

Anschließend erörterte Bach in verschiedenen Beiträgen die Wich-tigkeit der Pixexxmokokken für die Entstehung des Ulcus eorneae serpens, und er suehte auf experimeixtellem Wege die Frage nach der Zweck-mäßigkeit aseptischer oder antiseptischer Verbi $1 \frac{1}{8 d e}$ bei den geschwürigen Prozessen der Homhaxxt klarzxxstellexx.

Es folgten dann versehiedene Arbeiten zur Lehre von den Augen-muskellähnxungen, die für die Folgezeit Veraxxlassung wurden, daß Back der ganzen Frage nach der Innervation der äußeren $\mathrm{x} \pi \mathrm{xd}$ inneren Augen-muskxüatur mit besonderer Berücksichtigung ihres Kerngebietes systematisch nähertrat. Dieses Gebiet, dessen Bearbeitung gerade nicht zxi den einfachstexi geliört, hat ihn bis zxim Ende stets lebhaft interessiert xxnd w ird nicht behandelt werden können, ohne der grundlegenden, wenn axxclx nicht unbestrittenen Arbeiten Backs Erwähnung zxx tun. Denn zahlreiche Untersxxehungen speziell über die Kerne des N. oexxlomotorius legen Zeugnis ab von dem unermüdlichen Fleiße des Verblichenen. Seine eingehenden experimentellen xxnd klinischen Studien über die Гnnervationsbahnen der Irismuskulatur beim gesxuxden xxnd kranken Menschen, die er in ihrem experimentellen Teile nxit Hans Meyer zxx-sammen anstellte, haben, darüber kann kein Zvleifel seiix, der klinischen Forschung der organischen Erkrankungen des Gehirn- und Rücken-marks ein weites Xexxland eröffnet. Sein Lehrbuch der Pupillenlehre, in dem er inx Zusammenhange sehxe Erfahrungen niederlegte, wird jedem, der auf diesem Gebiete arbeitet, eirx unentbehrlicher Ratgeber sein, xxnd der Name Back bleibt stets verbunden mit dem Begriffe xxnd xxnserer Auffassxxng von der Bedeutung und deux klinischen Bilde der reflektorischen Pupi $1 / 8 n$ starre.

Die letzten Jahre widmete Bach denx iStudium entwieklungs-geschichtlicher Fragen, uxxd es war ihm ja noch vergönnt, das Erscheinen des seiner Feder entstammexxden ersten Teiles seines mit Seefelder zxx-sanxmen heraxxsgegebeixeix entwickluixgsgeschichtlichen Atlasses des menschlicheix Auges zxx erlebeix.

Fast zahllos sind die Arbeiten, die unter der Leituixg des ver-storbenen Meisters ausgeführt wurden, und die auf den versehiedensteix Gebieten der Axxgenheilkunde Aufklärung gebracht habeix. Seines Intex $3 / 4$ Sses xxnd seiner tatkräftigen Unterstützung war eben jeder sicher, der nxit dem Wunsche zu ihm kam, an seiner KJinik sich wissenschaftlich betätigeix zu dürfen. Dasselbe gait auch für seine engeren Schüler, seine Assistenten. Und wenn er, wie das axxclx selbstverstäixdlich ist, ein gewisses Maß wissenschaftlicher Tätigkeit als Bedingung aixsah für eiix gedeihlich.es Zusamnxenai $\cdot$ beiten, so ließ er andererseits jedexxx stets 
Ludwig Bach $\uparrow . \quad 567$

Gelegenheit, sich, ich inöchte sagen, wissenschaftlich auszuleben und sein Arbeitsgebiet in der Richtung zu suchen, die seinem besonderen per-sönlichen Geschmacke entsprach.

Der unerbittliche Tod riß Ludwig Bach mitten aus seinem Schaffen hinweg. So manclies noch ist im Entwurfe, so manches halbfertig zurückgeblieben, das vollendet reiche Früchte getragen hätte. Hoffen wir, daß sich der Nachfolger finde, dem es gelingt, das Be-gonnene zum guten Ende zu führen.

Was der Persönlichkeit Backs ihren besonders charakteristischen Stempel aufdrückte, waren seine hervorragenden Eigenschaften als Universitätsdozent. Es gab für ihn keine wichtigere Aufgabe, als die, seinen Schülern ein guter Lehrer zu sein; er empfand niemals eine größere Befriedigung als dann, wenn er den klinischen Unterricht ver-ließ mit dem Bewußtsein freudiger Genugtuung, daß ihm die Dar-stellung eines schwierigeren Kapitels der Augenheilkunde besonders gut gelungen war. Mit einem feinen Gefühl für die wechselnde Stimmung der Studierenden beseelt, war er stets bestrebt der besonderen Aufgabe gerecht zu werden, die jedem guten Dozenten am verlockendsten erscheinen muß, nämlich die Sprödigkeit der Materie in der Weise zu überwinden, daß er im steten, innigen, geistigen Konnex sich eins weiß mit seinen Hörern.

Schon $\mathrm{w}^{3} / 4$ hrend seiner Würzburger Dozentenzeit hatte Bach in seinen Ferienkursen die große Ereude und Genugtuung, ein zahlreiches Auditorium zu seinen Füßen zu sehen, und mancher seiner damaligen Hörer wird sich noch erinnern, wie er es verstand, die nicht selten vorhandenen Lücken im ophthalmologischen Wissen der Examenskandi-daten auszufüllen, und wird mit Dankbarkeit der Hilfe eingedenk bleiben, die er in Examensnöten von ihm erfahren. Bach war keiner von denen, die mit angeborener Souveränität den Lehrstoff beherrschen wollen. Dieser Umstand und sein gewissen-haftes Bestreben, seinen Schülern stets das Beste zu geben, brachten es mit sich, daß er sich stets peinlich auf den klinischen Unterricht vor-bereitete; was er gab, war dann aber auch wie aus einem Guß.

Seine frische Art zu dozieren und sein oft hinreißender Humor hielten sein Auditorium in fortgesetzter Spannung trotz der, zumal im heißen Sommer, wenig zum Unterricht geeigneten Zwölfuhrstunde und der schon mehr oder weniger vorhandenen Ermüdung der Hörer.

Er sparte aber auch weder Mühe noch Kosten, wenn es gait, einen besonders interessanten Fall für den Unterricht zu gewinnen, und es war dann oft überraschend, was er aus einem Falle zu machen wußte, urn ihn mit alien seinen interessanten Einzelheiten dem Verständnisse seiner Hörer nahe zu bringen.

Ein schönes Denkmal ihrer Verehrung und Zuneigung haben ihm die Marburger Klinizisten in den wenigen, aber inhaltreichen Worten gesetzt, die sie ihm öffentlich widmeten: „Vielen Geschlechtern von jungen Medizinern war er ein trefflicher Führer, der mit klarer Stetig-keit unser Wissen zu sicherem Besitz formte. Sein Leben erfüllte sich in der herben Tragik ärztlicher Pflicht. So steht sein Bild vor uns als

Zeitschrilt für Augenheilkunde. Bd. XXVII. Heft 6. 39

568

Ludwig Bach $\uparrow$.

hohes Ziel unserer Nacheiferung. Tiefe Dankbarkeit macht sein An-denken bei uns unvergänglich. “

Ein Mann von den Eigenschaften des Verblichenen mußte auch ein tüchtiger Arzt sein, und das Andenken, das Ludwig Bach in den Herzen seiner Kranken zurückließ, wird dauernder sein als sein Grabmal von Stein und Erz. Das Wohl seiner Patienten ging ihm über alles, und er nahm 
geradezu persönlichen Anteil an einem jeden, wohl das höchste Lob für einen vielbeschäftigten Dozenten und Kliniker.

Es fiel ihm nicht leicht, die vollendete technische Geschicklichkeit zu erlangen, die

Glücklicheren oft angeboren ist; allein er verstand es, durch stete Uebung das Mangelnde zu ersetzen, und wurde ein ausge-zeichneter Operateur, der alien Eventualitäten gewachsen war. Und wenn der Erfolg schließlich das Maßgebende ist, er hatte vorzügliche Result ate bei seinen Operationen, speziell beim Altersstar und, was nicht jeder Operateur von sich sagen kann, auch eine glückliche Hand.

So begleitet denn eine große Zahl erfolgreich behandeiter, dank-barer Patienten den Heimgang des geschätzten Arztes mit tiefer Teil-nahme.

Diese seine Eigenschaften verbanden sich mit einer offenkundigen organisatorischen Befähigung, die ihre ziffernmäßige Anerkennung in einem bedeutsamen Ansteigen der Frequenzkurve der ihm unterstellten Klinik fand: während seiner nicht ganz 12 Jahre umfassenden Direk-torialzeit ging sie auf mehr als die doppelte Höhe hinauf.

Nicht minder herzlich trauern ihrem dahingeschiedenen Kollegen die Aerzte der Praxis nach, mit denen ihn berufliche Bande verknüpften. Er führte in letztem Jahre den Vorsitz im Marburger ärztlichen Verein und bewies nicht nur für dessen wissenschaftliche und Standesaufgaben, sondern auch für die Notlage des ärztlichen Berufes ein voiles Ver-ständnis, das er stets bereit war durch Rat und Tat zu beweisen.

Es würde, wie ich glaube, wenig der im tiefsten Grunde doch mehr aufs einfache gerichteten Sinnesart des Verstorbenen entsprechen, wollte ich hier öffentlich das Lob seiner rein menschlichen Vorzüge singen. Nicht selten bleibt ja auch die innerste Charaktergrundlage eines Menschen trotz aller seiner Offenherzigkeit mehr oder weniger verborgen.

Nur eines möchte ich betonen, das sich wie ein breites Band durch das Lebensschicksal des Dahingegangenen zog, das war ein ihm inne-wohnender, selten starker Drang nach wissenschaftlicher Betätigung, das war eine Arbeitslust und Arbeitsfreude, die ihn selbst auf dem Krankenlager nicht verheß.

Im übrigen werden seine persönliche Liebenswürdigkeit und sein Entgegenkommen in alien Dingen stets dankbarer Anerkennung gewiß sein; sein frischer, gesunder Humor, der sich oft und zumal dann, wenn er seine eigene Person betraf, zum Sarkasmus wandeln konnte, verließ ihn nur selten. Ein lebhaftes Bedürfnis nach geselligem Verkehr be-herrschte ihn, und seine sich oft äußernde gesunde Lebensauffassung war stets bereit, das ,,Nil humani a me alienum" in die Tat umzusetzen. Bei aller Einfachheit der Sitten versagte er doch auch den kleinen Unfall- und Versicherungskunde.

569

Freuden des menschlichen Daseins die Bereclitigung nicht; kein Wunder, daß der Verstorbene auch rein menschlicli vielen nahe stand.

So steht denn das Bild Ludwig Bachs vor unserem geistigen Auge als das einer Persönlichkeit, deren Spuren nicht verwehen, solange die Wissenschaft sich dankbar ihrer Jünger erinnert. Durch seine Verdienste als Forscher, Lehrer und Arzt hat er sich ein unvergängliches Denkmal gesetzt. Sein Leben währte zwar keine 70 Jahre, aber es ist schön und köstlich gewesen, denn es war Mühe und Arbeit. Ehre seinem Andenken!

$-\beta>\alpha \% \beta$-Marburg.

Unfall- und Versicherungskunde.

Prof. Dr. P/afe-Düsseldorf: Die Spruehpraxis des Reichsversicherungs-amts bei Augenverletzungen und Sehstörungen. Stuttgart bei Ferdinand Enke. 28 S. Preis 1,20 M. 
Die Veröffentlichung ist gekürzt als Vortrag auf der Versammlung Rheinisch-Westfälischer Augenärzte in Düsseldorf am 10. II. 1912 ge-halten.

Verf. beschränkte sich auf Mitteilung solcher Entscheidungen des Reiehsversicherungsamts, denen eine gewisse grundsätzliche Bedeutung, wenn auch nur für die Gegenwart, innewohnt. Er halt ihre Kenntnis und Beachtung von seiten des Gutachters für nützlich, urn nicht ohne Vorbehalt Renten zu empfehlen, die mit Erfolg von der einen oder anderen Partei, dem Versicherten oder dem Versicherungsträger, ange-fochten werden können. Er weist aber andererseits mit Recht darauf hin, daß diese Entscheidungen keine Dogmen sind und sein können. Der fortschreitenden Entwicklung unserer Kenntnisse von den Be-ziehungen der Körperfunktionen zur Erwerbsfähigkeit wird auch die Spruehpraxis Rechnung tragen müssen, sobald schlüssige neue Ergeb-nisse vorliegen. Schon aus diesem Grunde wird jeder ärztliche Gut-achter von Erfahrung sich das Recht wahren müssen, nach eigenem besten Wissen und Können an Hand seiner eigenen Erfahrungen in jedem Falle seine Ansicht auszusprechen, auch wo sie mit der Spruehpraxis nicht übereinstimmt. Verf. halt dies sogar für Pflicht des ver-sierten Gutachters und hofft, daß bei verständnisvoller Mitwirkung der Aerzte „die Rechtsprechung von dem bisher noch sehr breiten Boden der Theorie immer mehr auf den der praktischen Lebenserfahrung liinübergehen werde, ein Fortschritt, der auch im Interesse sozialer und sittlicher Hygiene liegt.

Im einzelnen behandelt Verf. ganz kurz durch Zitate aus Reichs-versicherungsamtsEntscheidungen die Hauptpunkte aus der Gutachter-praxis und erläutert sie mit kritischen Bemerkungen.

Besprochen wird: 